\title{
Cell Image Analysis: Algorithms, System and Applications
}

\author{
Takeo Kanade ${ }^{1}$, Zhaozheng Yin ${ }^{1}$, Ryoma Bise ${ }^{1}$, Seungil Huh ${ }^{1}$, Sungeun Eom ${ }^{1}$, Michael F. Sandbothe ${ }^{1}$ \\ and Mei Chen ${ }^{2}$ \\ ${ }^{1}$ Carnegie Mellon University, ${ }^{2}$ Intel Labs Pittsburgh
}

\begin{abstract}
We present several algorithms for cell image analysis including microscopy image restoration, cell event detection and cell tracking in a large population. The algorithms are integrated into an automated system capable of quantifying cell proliferation metrics in vitro in real-time. This offers unique opportunities for biological applications such as efficient cell behavior discovery in response to different cell culturing conditions and adaptive experiment control. We quantitatively evaluated our system's performance on 16 microscopy image sequences with satisfactory accuracy for biologists' need. We have also developed a public website compatible to the system's local user interface, thereby allowing biologists to conveniently check their experiment progress online. The website will serve as a community resource that allows other research groups to upload their cell images for analysis and comparison.
\end{abstract}

\section{Introduction}

Advances in optics and imaging systems have enabled biologists to visualize a living specimen's dynamic processes by time-lapse microscopy images. However, the image data recorded during even a single experiment may consist of hundreds of objects over thousands of images, which makes manual inspection a tedious and inaccurate option. This paper addresses the need for computational tools and community resources that can enable the quantitative system-level biology automatically. The domain of our current interests are cell image analysis algorithms including microscopy image restoration, cell mitotic event detection and cell tracking by data association, which are integrated into an online automated system. Figure 1 shows one of our system's capabilities. In a stem cell manufacturing experiment, the number of cells grew from $20+$ to hundreds in about 55 hours (Figure 1(a-b)). We detect and track each individual cell's movements in space and time, and generate corresponding lineage trees (i.e. mother-daughter relationship) to compute metrics for biologists to measure the cell proliferation process. For example, Figure 1(c) shows tracking trajectories of three cell families and Figure 1(d)

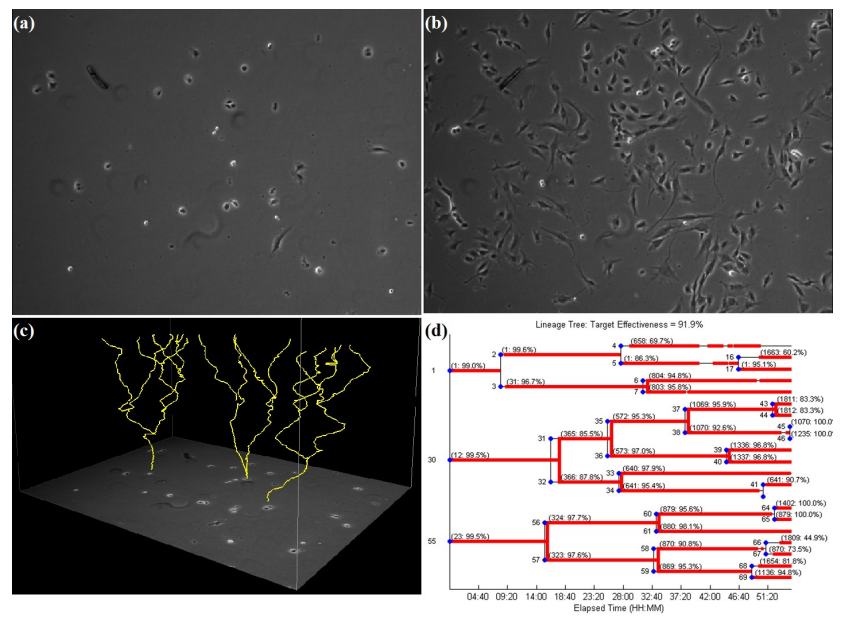

Figure 1. Generating cell lineage trees. (a) Beginning of the experiment with 20+ cells; (b) End of the experiment (55 hours later) with hundreds of cells; (c) Trajectories of three cell families shown in space and time; (d) Lineage trees and performance evaluation (thin black lines: three human annotated lineage trees; overlaid thick red lines: correctly-tracked cells by our system).

shows their lineage trees compared to the human annotated ones.

\section{Related literature}

As recently reviewed in $[6,14,17]$, tracking and analyzing cell movements are of particular interest to many living cell experiments. There are three main categories of existing tracking methods: filtering-based sampling approaches, model-based evolution approaches and detection-based association approaches.

Particle filtering is a filtering-based approach widely used for multiple object tracking. In [19], multiple models such as measurement models and dynamic models need to be known before estimating the posterior distributions of an object's current states. Gating and hierarchy searching techniques are used to reduce the large computational cost introduced by Monte-Carlo sampling $\left(10^{3}\right.$ particles are used per object per frame). Human interaction or other initialization methods are required to define the prior distributions of object states in the first frame. 
The idea of model-based tracking is to create and update a model for each object to be tracked. For example, a coupled mean-shift algorithm is developed to track migrating cells in phase-contrast microscopy images [4], where human operators are asked to select cells to be tracked and also initialize the object appearance models at the beginning. Mean-shift algorithms are then performed on the preprocessed images (thresholded, equalized and masked images). This method works well if the cell shape used to define multiple mean-shift kernels is known and cells move relatively slowly between consecutive frames. Another option of model-based approach is the active contour method that is extensively researched ([5] and references therein). Though the active contour method handles the cell shape deformation well and uses object contours of the previous frame as initializations in the current frame, reinitialization is usually required if objects show large displacement between frames or new object appearing events happen.

The strategy of detection-based association approach is to segment and locate objects first and then associate those objects/tracklets (short accurate object trajectories) among frames [6, 14]. Some popular segmentation methods employed in the previous cell-tracking systems include intensity thresholding [1], gradient (edge) detection [7], morphological operations [8] and watershed algorithms [20]. However, due to intensity variations, image noise and artifacts from the optical system (e.g. halo in the phase contrast microscopy images), intensity thresholding is usually errorprone. Edge detection is more robust than thresholding, but it does not work well on low-contrast images or flat cell regions. Morphological operations only provide rough segmentation and the watershed approach usually suffers from image noise and tends to over-segment images.

Mitosis, or cell division, is one important phenomenon we should consider in cell tracking systems. A series of distinctive changes during mitosis (e.g. the changes of object appearance and shape) encumbers model-based tracking methods. Indeed, accurate detection of mitosis events can benefit frame-by-frame or tracklet-by-tracklet association. Recently, several mitosis event detection algorithms that are independent of tracking results have been proposed. Li et al. [9] adopted a cascade Adaboost algorithm on volumetric Haar-like features to detect mitosis events. The exhaustive search based on a sliding window scheme is computationally expensive and volumetric Haar-like features are not good at capturing long-term temporal dynamics. Liu et al. [12] applied the Hidden Conditional Random Field (HCRF) model to detect patch sequences containing mitosis. This work does not provide information on when mitosis is completed and daughter cells are born, which indicates when a trajectory of a mother cell divides into two trajectories of its daughter cells. In addition, the overall approach is computationally expensive due to its preconditioning step.
Based on detected cell candidates, linear programming [1, 8] and min-cost flow [15] were applied to match objects across frames. The frame-by-frame association approach works well only if objects are accurately detected in each frame. However, cells often touch and partially overlap each other and form a cell cluster with blurry intercellular boundaries. Such clusters cause difficulty in the above association approaches, often resulting in loss of cell tracks and confusion of cell identities.

\section{Our work}

To address the drawbacks of the previous approaches, we introduce three algorithms for cell image analysis that are integrated into an online automated system:

1. We present a microscopy image restoration and segmentation method based on the specific microscopy imaging model. A quadratic optimization function with sparseness and smoothness regularizations is formulated to restore the "authentic" microscopy images without artifacts such as halo and shading. With artifacts removed, high quality segmentation can be achieved by simply thresholding the restored image.

2. We propose a three-step approach for mitosis detection: candidate patch sequence construction, feature extraction, and identification of mitosis occurrence and localization of birth event (the time and location at which the two daughter cells first appear and the boundary between them is clearly shown). In the first step, the search space is reduced from the entire video volume to small-size candidate patch sequences. In the second step, visual features from each candidate patch are extracted based on the characteristics of microscopy cell images. In the last step, we determine whether each candidate patch sequence contains a birth event and detect the spatial and temporal locations of all birth events.

3. Based on the segmentation and mitosis detection results, we propose a cell-blob association approach tolerant of missing cell detection, cell overlapping and dividing. The tracking system is automatically initialized by the segmentation algorithm and can handle objects coming into or leaving the field of view.

\section{Overview of Our System}

Figure 2 shows an overview of the integrated system. Researchers can upload cell images from their database to our file server, capture microscopic images of living cells in real time and upload them, or retrieve image analysis results to check their experiment progress. Once microscopy images are uploaded to the file server, the computing clusters run cell image analysis algorithms in parallel to process images and output results to the file server. The file server stores all image files and processed results (segmentation mask, mitosis detection, tracking trajectories and cell met- 


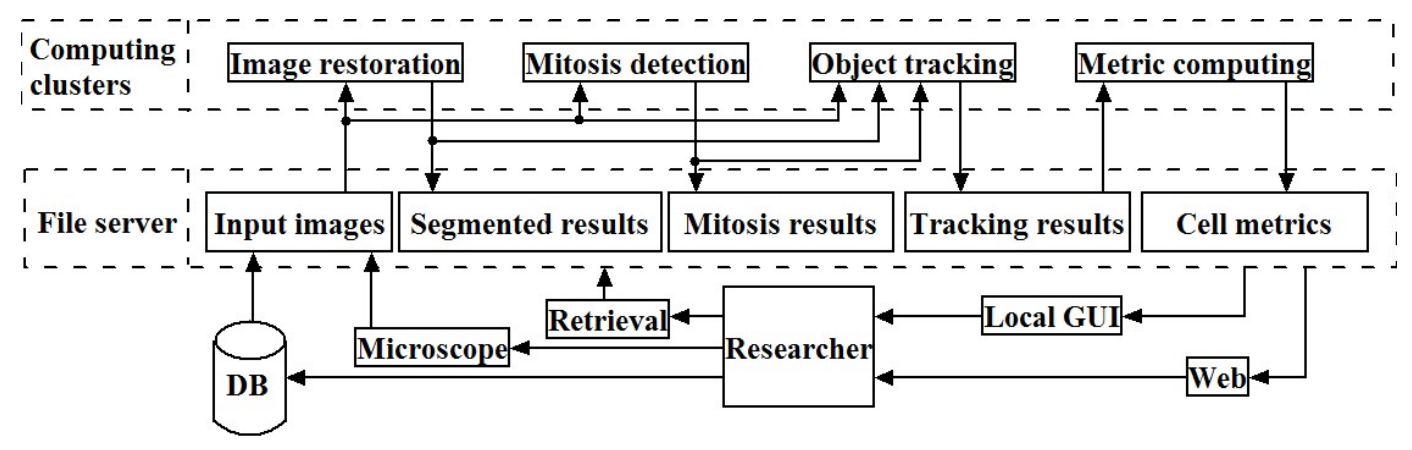

Figure 2. Overview of our system.

rics), which can be viewed in a local graphical user interface (GUI) or through our website.

Our system supports online biological experiments and we have successfully tested it worldwide. After biologists started culturing cells in their lab overseas, microscopy imaging system captured and uploaded the images to our file server periodically. Our system processed the images in real-time and provided corresponding metrics to biologists for adaptive experiment control. Biologists can check their experiment progress conveniently (e.g. from a mobile device with internet connection), without having to wait in their lab for hours.

\section{Algorithms and Performance Evaluation}

In this section, we first describe the three cell image analysis algorithms in the integrated system and then quantitatively evaluate the system's performance.

\subsection{Microscopy Image Restoration}

Based on the principle of detection-based tracking approach (i.e. first detect, then track), segmenting object candidates from the background is fundamental to the tracking system. Figure 3(a) shows a typical phase contrast image where cells are surrounded by bright halos, some cell structures (e.g. nuclei and cell membrane) consist of multiple dark regions, and cellular fluid inside the membrane has similar intensity as the background. This presents a lot of challenges to traditional image segmentation techniques. Instead, we attack the segmentation problem by considering the unique characteristics of microscopy image formation process such as the halo artifacts of phase contrast images. Using the recently discovered microscopy imaging model [21], we formulate a regularized quadratic cost function to restore the artifact-free microscopy images:

$$
\begin{array}{r}
\mathbf{O}(\mathbf{f})=\|\mathbf{H} \mathbf{f}-\mathbf{g}\|_{2}+ \\
\omega_{l} \mathbf{f}^{T} \mathbf{L f}+\omega_{s}\left\|\boldsymbol{\Lambda}_{\mathbf{s}} \mathbf{f}\right\|_{1}+\omega_{t}\left(\mathbf{f}-\mathbf{f}_{t}\right)^{T} \boldsymbol{\Lambda}_{t}\left(\mathbf{f}-\mathbf{f}_{t}\right)
\end{array}
$$

where $\mathbf{f}$ is the vectorized image to be restored such that background pixels have uniform zero values and foreground pixels have positive values, based on which simple thresholding method can work well for the segmentation task. $\mathbf{H}$ is the imaging model in a matrix format from [21], $\mathbf{g}$ is the vectorized observed image. The first regularization term in Eq. 1 is spatial smoothness where $\mathbf{L}$ is a Laplacian matrix defining the spatial intensity similarity between neighboring pixels such as the one used in [11]. The second is a $l_{1}$ sparsity term where $\boldsymbol{\Lambda}_{\mathbf{s}}$ is a diagonal matrix penalizing large f values. The last regularization term is to maintain the temporal consistency between consecutive images where $\boldsymbol{\Lambda}_{t}$ is a diagonal matrix defining the intensity similarity between neighboring pixels in the temporal domain and $\mathbf{f}_{t}$ is the restored image in the previous frame. The optimal weights for the three regularization terms $\left(\omega_{l}, \omega_{s}\right.$, and $\left.\omega_{t}\right)$ are learned from training images by grid-search.

The regularized objective function can be minimized using a nonnegative updating scheme [18]. Figure 3(b) shows the restored image where a simple thresholding method is enough to separate the cell pixels from background pixels.

Differential Interference Contrast (DIC), another popular microscopy imaging technique, displays a pseudo threedimensional shading effect and cell intensities are quite similar to background pixels as shown in Figure 3(c). Similarly, based on the DIC imaging model [10], we can restore an image conducive to thresholding (Figure 3(d)).

\subsection{Mitosis Detection}

We propose a three-step mitosis detection approach: patch sequences construction, feature extraction and birth event location. We detect candidate patch sequences based on intensity change, since cells typically exhibit increased brightness during mitosis events (Figure 4(a)). More specifically, after detecting small bright rectangular regions in each image using thresholding and convolution, overlapped regions are combined into one patch. Then, spatiallyoverlapped patches across consecutive frames are collected into a candidate patch sequence.

For each patch in candidate patch sequences, we compute unique-scale gradient histograms as visual features. These features are similar to SIFT [13] in that gradient his- 


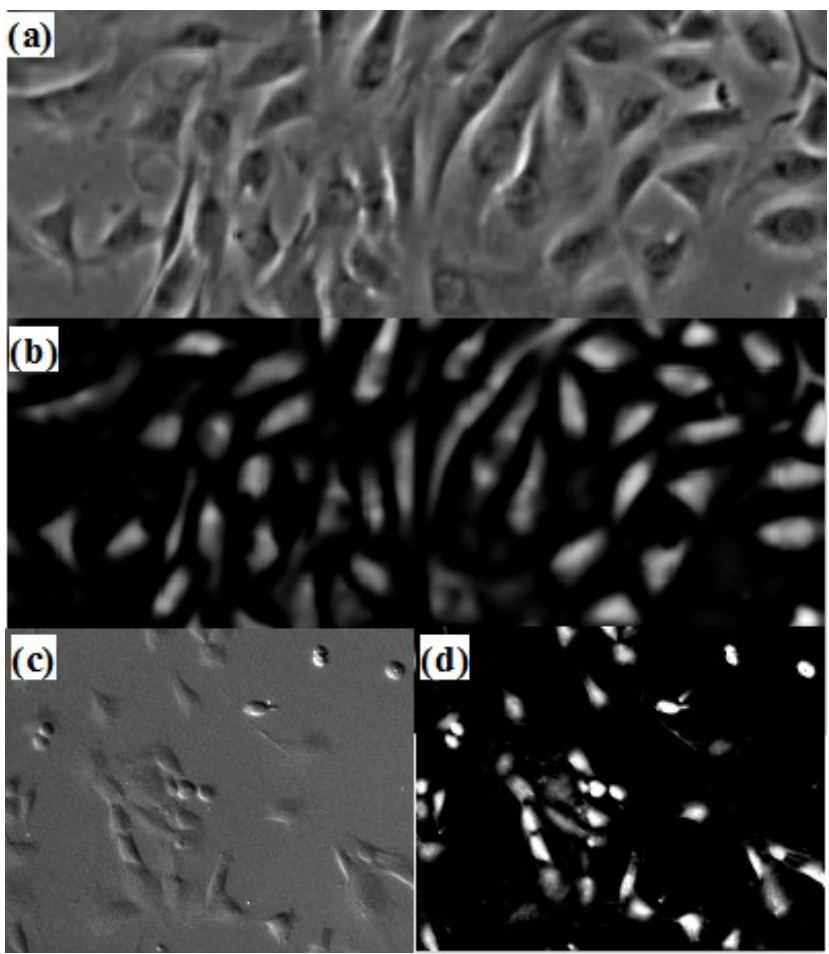

Figure 3. Microscopy image restoration. (a,b): Input and restored phase contrast images; (c,d): Input and restored DIC images.

tograms are computed; however, we fixed the scale since the distance between the microscope lens and cells are almost uniform. In addition, to obtain similar features regardless of a cell's orientation, we duplicate patch sequences in the training set by rotating all patches in the sequence along several different orientations. This rotation invariance scheme shows more reliable results compared to the original SIFT rotation invariance scheme with the major axis.

After the first two steps, we will decide whether each candidate patch sequence contains a birth event and in which frame the birth event is located. For the first decision task, we adopt the HCRF model [16]. HCRF model has an advantage in that it can model temporal dynamics in patch sequences containing different numbers of patches. For the second decision task, we trained a version of Support Vector Machine (SVM) that outputs probabilities. For training, patches containing a manually annotated birth event are used as positive samples and other patches in patch sequences that do not contain mitosis are negative samples. For each patch sequence determined to include a mitosis event, the SVM is applied and the patch with the highest probability is decided to contain a birth event. Figure 4(b) shows the mitosis detection results (yellow rings) of two cell families by our mitosis detection algorithm. The cell branch trajectories are well linked by applying the birth event information in the following association approach.
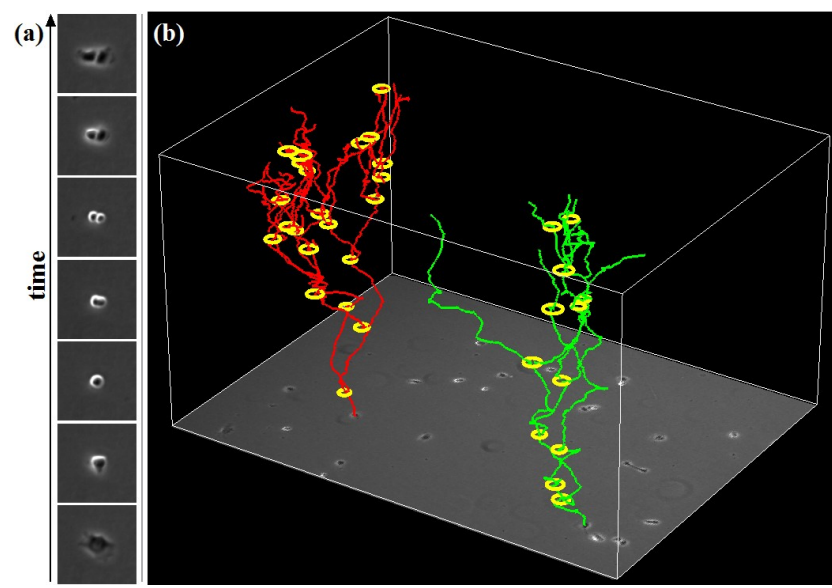

Figure 4. Mitosis detection. (a) A patch sequence showing a cell's appearance change during a mitosis event; (b) Mitosis detection is used in association-based tracking module (yellow rings: detected mitosis events around tree bifurcations, red and green trees: two well-tracked cell families viewed in the spatiotemporal space).

\subsection{Cell Tracking by Association}

The image segmentation algorithm segments blobs from input images that can be individual cells or cell clusters (overlapped cells), and the mitosis detection algorithm locates birth events where and when one cell divides into two cells. Based on the outputs of these two algorithms, we developed a cell-blob correspondence algorithm performing data association between the cells in the previous frame and the blobs segmented in the current frame. In detail, the association algorithm makes the following hypotheses with corresponding likelihood for all possible cell actions (Figure $5(\mathrm{a}))$ :

(1) one-to-one hypothesis: a cell migrates to a new position:

$$
p_{1 \rightarrow 1}\left(c_{i}, b_{j}\right)=e^{-\frac{\left\|f\left(c_{i}\right)-f\left(b_{j}\right)\right\|}{\sigma}}
$$

where $c_{i}$ represents the $i$ th cell in the previous frame and $b_{j}$ represents the $j$ th blob in the current frame. $f(\cdot)$ computes an object's feature vector where different types of features can be incorporated such as appearance histogram, shape and motion history. $\sigma$ is a free parameter to adjust the distribution. The likelihood of a one-to-one hypothesis, $p_{1 \rightarrow 1}\left(c_{i}, b_{j}\right)$, is computed based on the distance between two feature vectors. We apply a gating region to reduce the size of one-to-one hypothesis set such that cell $c_{i}$ only looks for its corresponding blob around its local area. When there is no nearby blob in the current frame (missing detection case), cell $c_{i}$ will search its corresponding blob in the next several frames (i.e. within a sliding temporal window).

(2) one-to-none hypothesis: a cell exits from the field of view:

$$
p_{1 \rightarrow 0}\left(c_{i}\right)=e^{-\frac{d\left(c_{i}\right)}{\lambda}}
$$

where $d\left(c_{i}\right)$ computes the spatial distance between the cen- 
troid of cell $c_{i}$ and the image boundary. $\lambda$ is a free parameter to adjust the distribution. Only cells near the image boundary have one-to-none (exit) hypotheses.

(3) none-to-one hypothesis: a blob enters the field of view:

$$
p_{0 \rightarrow 1}\left(b_{j}\right)=e^{-\frac{d\left(b_{j}\right)}{\lambda}}
$$

Similar to one-to-none hypothesis, only blobs near the image boundary have none-to-one (enter) hypothesis.

(4) one-to-two: a cell divides into two cells:

$$
p_{1 \rightarrow 2}\left(c_{i}, b_{j_{1}}, b_{j_{2}}\right)=e^{-\frac{\left\|f\left(c_{i}\right)-f\left(b_{j_{1}}, b_{j_{2}}\right)\right\|}{\sigma}}
$$

Only cells near the spatiotemporal location of a birth event have one-to-two (mitosis) hypothesis. To handle missing blob detection or inaccurate birth event detection, we use a sliding temporal window around the detected mitotic moment, i.e. a mother cell looks for its two daughter blobs within several succeeding frames. To compute the feature vector of two separated blobs, $f\left(b_{j_{1}}, b_{j_{2}}\right)$, we translate $b_{j_{1}}$ and $b_{j_{2}}$ towards each other until they overlap, and then extract the feature vector over the joint blob.

(5) many-to-one: multiple cells overlap:

$$
p_{n \rightarrow 1}\left(c_{i_{1}}, \ldots, c_{i_{n}}, b_{j}\right)=e^{-\frac{\left\|f\left(c_{i_{1}}, \ldots, c_{i_{n}}\right)-f\left(b_{j}\right)\right\|}{\sigma}}
$$

When several cells are close to each other in the previous frame and a nearby large blob is detected in the current frame, those cells have a many-to-one hypothesis. We shift the multiple cells to form a joint cell to extract feature vector $f\left(c_{i_{1}}, \ldots, c_{i_{n}}\right)$. When tracking the overlapped blob in the succeeding frames, we apply a contour-matching method [2] to separate it into its member cells, thus the cell identities are maintained. Figure 5(b) shows an example of tracking overlapped cells with many-(cells)-to-one(blob) hypothesis where multiple cells partially overlap and the blob contour of the resultant cluster is comprised of partial contours of its member cells.

After computing the likelihood of all the $M$ hypotheses over $N_{1}$ cells and $N_{2}$ blobs, we get the $M \times 1$ likelihood vector $\mathbf{p}$ and formulate the following optimization problem

$$
\arg \max _{\mathbf{x}} \mathbf{p}^{T} \mathbf{x}, \quad \text { s.t. } \mathbf{C}^{T} \mathbf{x} \leq \mathbf{1}
$$

where $\mathbf{x}$ is a $M \times 1$ binary vector, and $\mathbf{x}_{k}=1$ means the $k$ th hypothesis is selected in the global optimal solution. $\mathbf{C}$ is a $M \times\left(N_{1}+N_{2}\right)$ matrix, each row of which denotes which object IDs are involved in that hypothesis. The constraint $\mathbf{C}^{T} \mathbf{x} \leq \mathbf{1}$ guarantees that each object ID appears in no more than one hypothesis in the optimal solution, i.e. no conflict hypotheses in the solution. Figure 5(c) shows a simple example with $M=8, N_{1}=3$ and $N_{2}=3$. In the optimal solution, blobs 4 and 5 will be assigned with new cell IDs since they are newborn daughter cells of mother cell 7, and blob 6 will have two member cells with inherent cell ID 8 and 9.
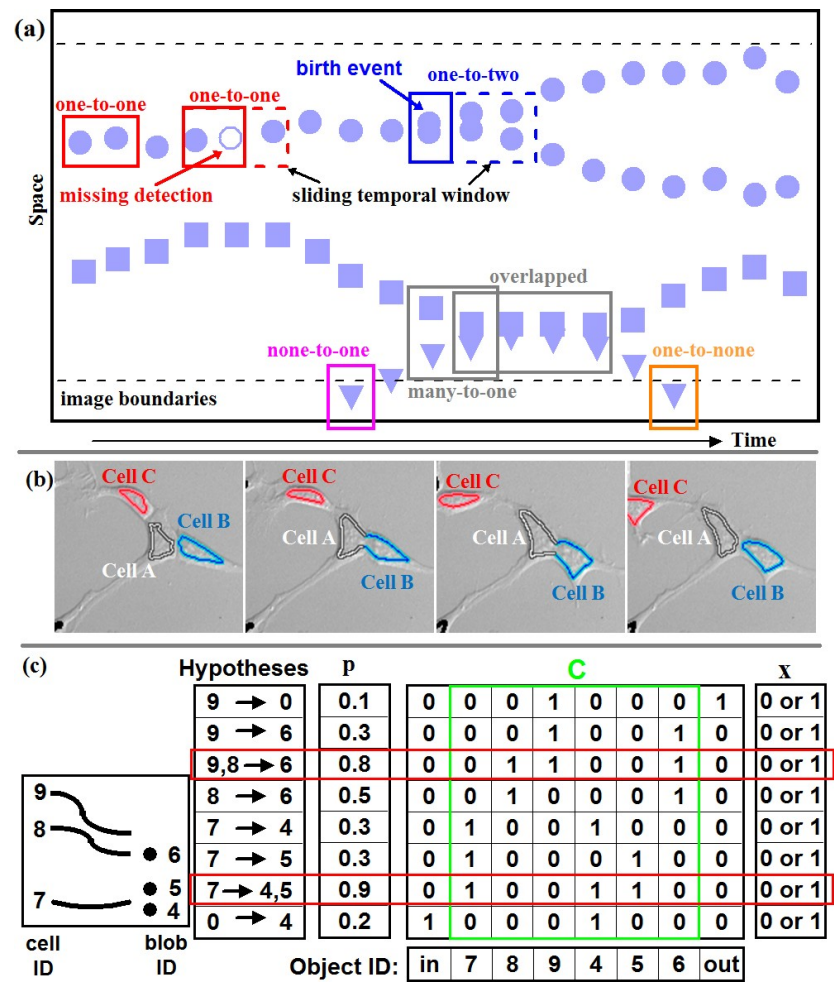

Figure 5. Tracking by association. (a) Cell-blob hypotheses; (b) Separate overlapped cells by matching partial contours during many-to-one hypothesis; (c) An integer programming example where the optimal solution is highlighted by red.
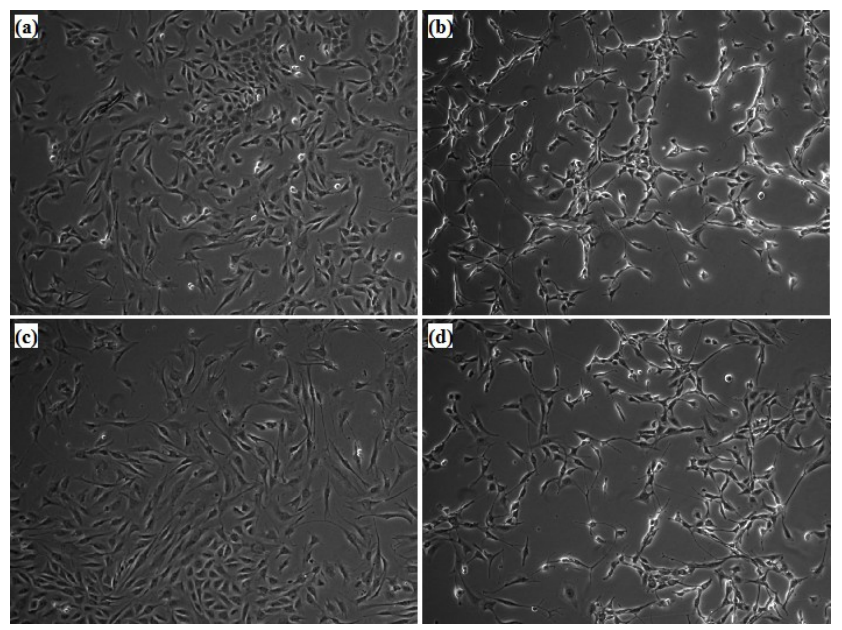

Figure 6. Sample images from four cell culture conditions. (a) Control; (b) With FGF2 medicine; (c) With BMP2 medicine; (d) With FGF2+BMP2 medicine.

\subsection{Performance Evaluation}

We recorded 16 image sequences under four different cell culture conditions with sample images shown in Figure 6 . The images are captured every 5 minutes and each sequence consists of 1000 images with the resolution of 

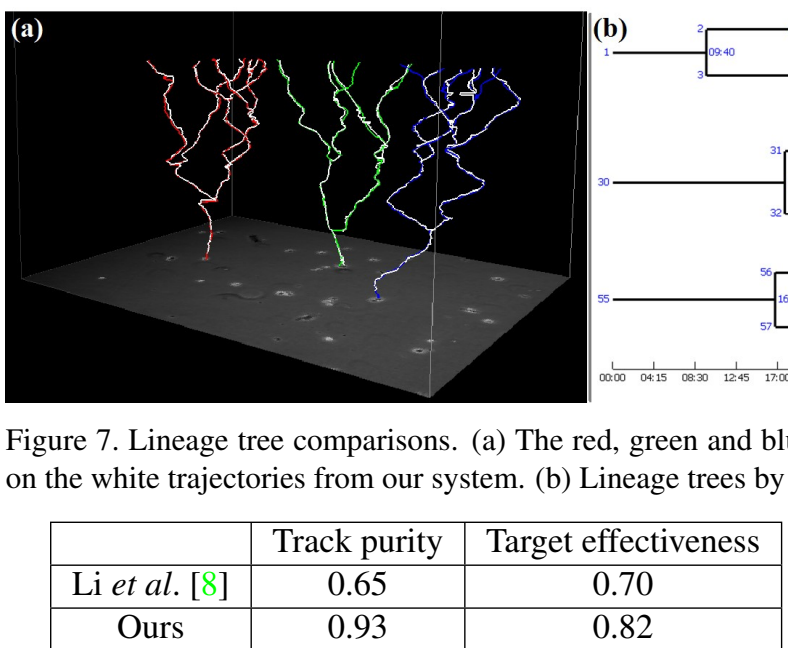

Table 1. Comparison of our system with [8] on a sequence with all cells annotated.

$1392 * 1040$ pixels. The total number of annotated cells in the 16 sequences is $135859^{1}$.

Figure 7(a) shows an example of the computer-tracked trajectories (white) compared to the human-annotated ones (red, green and blue) in spatiotemporal space. Figure 7(b) and (c) show the binary lineage trees by human and our system, respectively. The tree comparison is a strict evaluation criterion. We compare not only the spatial distance between human-annotated cell locations and computertracked ones, but also the mother-daughter relationships between tree branches. Visually comparing Figure 7(b) and (c), we would conclude that the two sets of lineage trees are matched well overall. Though there is a missing cell detection and birth event detection on cell 1 in Figure 7(c), its daughter cells are detected and tracked later, thus the sublineage trees are recovered.

We use two quantitative criteria to assess the system performance: track purity and target effectiveness [3]. The track purity is defined as the number of track observations (computer-generated) matched to the best target (human annotated) over the total number of track observations. It indicates how many observations from a computer-generated track are from the correct target. Similarly, we define target effectiveness as how well targets are followed by tracks.

As shown in Table 1, our system achieves higher track purity and target effectiveness than the state-of-the-art method in [8] on the full-annotated sequence (Exp1 under BMP2 condition). Table 2 shows the target effective-

\footnotetext{
${ }^{1}$ Initially, we planned to annotate every cell in each image for all the sequences. However, it took a senior PhD student from the Bioengineering department one month to annotate one sequence from the first image to the 780th image. He could not continue beyond that because cells are highly confluent after then. Since it is too time-consuming to annotate the migration and mitosis histories for all cells by human, for all the other 15 sequences, we randomly pick three cells at the beginning of each sequence and annotate the three cells' family trees through the 780th image.
}
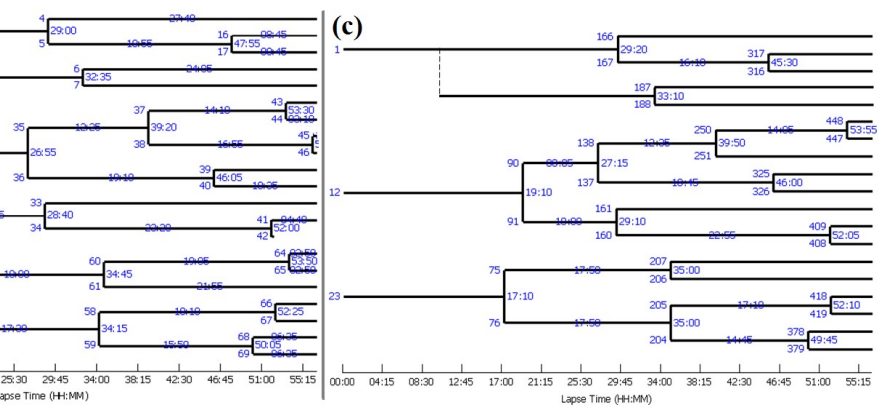

trajectories that are human annotation of three cell families are overlaid annotation; (c) Lineage trees from our system.

\begin{tabular}{|c|c|c|c|c|c|c|}
\hline Cond & Alg & Exp1 & Exp2 & Exp3 & Exp4 & Avg \\
\hline \multirow{2}{*}{ Control } & {$[8]$} & 0.69 & 0.70 & 0.76 & 0.65 & 0.70 \\
\cline { 2 - 7 } & Ours & 0.84 & 0.85 & 0.82 & 0.81 & 0.83 \\
\hline \multirow{2}{*}{ FGF2 } & {$[8]$} & 0.56 & 0.67 & 0.57 & 0.48 & 0.57 \\
\cline { 2 - 7 } & Ours & 0.62 & 0.66 & 0.67 & 0.60 & 0.64 \\
\hline \multirow{2}{*}{ BMP2 } & {$[8]$} & 0.70 & 0.71 & 0.61 & 0.55 & 0.63 \\
\cline { 2 - 7 } & Ours & 0.82 & 0.88 & 0.72 & 0.77 & 0.80 \\
\hline FGF2+ & {$[8]$} & 0.68 & 0.68 & 0.70 & 0.78 & 0.71 \\
\cline { 2 - 7 } BMP2 & Ours & 0.80 & 0.76 & 0.78 & 0.82 & 0.79 \\
\hline
\end{tabular}

Table 2. The target effectiveness comparison between our system and [8] on 16 experiments of four cell culturing conditions.

ness comparison on all the 16 sequences $^{2}$. On average, we achieve around $80 \%$ target effectiveness of three culture conditions except the FGF2 case, compared to $66 \%$ of [8]. When computing the target effectiveness, a target in the lineage tree is considered as not being tracked if: the object centroid from the track observation deviates from the human annotated centroid larger than $T$ pixels (e.g. $T=10$ ); there is no corresponding track observation for a period larger than the sliding temporal window (accumulated missing detection); or the cell ID of the track observation switches to another track (wrong lineage tree relationship). In the FGF2 culturing condition (Figure 6(b)), cells deform largely with irregular shape and heavily overlap each other, which makes the tasks of segmentation, mitosis detection and tracking harder. The future work to further improve our system's performance will include trajectory pruning and cell-blob affinity model learning.

\section{Local GUI and Website}

To handle the heavy computation load of many biological experiments at the same time, we use a cloud computing cluster and a virtual machine manager. As shown in Figure 2, the cell image analysis algorithms run on the computing clusters and output results onto a file server. To visualize the system's output, we implement a local GUI in MATLAB and utilize it in two ways: one is for biologists to

\footnotetext{
${ }^{2} \mathrm{We}$ are not able to compute track purity for the 15 partially-annotated sequences because it needs all cells to be annotated while we have three cell families annotated per sequence so far.
} 
(a)

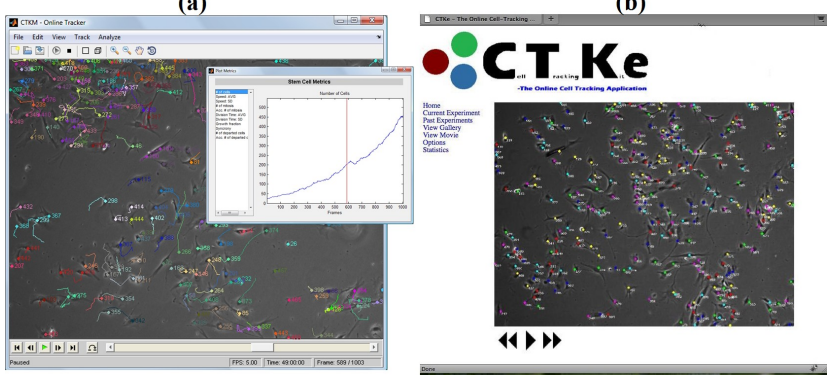

Figure 8. (a): Local stand-alone GUI; (b): Public website.

check their experiment progress, and the other is for algorithm developers to debug their programs. The local GUI is synchronized with the file server. Once a new biology experiment is started and images are captured, uploaded and processed, all the results (segmentation, mitosis detection, tracking and cell metrics) can be visualized in the GUI. A screen shot of the GUI is shown in Figure 8(a). In the main window, segmented cells are highlighted together with trajectories shown on the input image. The trajectories are useful to observe how cells behave and can be displayed in 3D. Various cell metrics computed from cell lineage trees (e.g. the number of cells, division time, growth fraction, etc.) can be visualized in a pop-up window in real time. The GUI also provides an offline mode during an online experiment so that a user can review the results in the previous frames.

Compatible to the local GUI, we also developed a web application. The web interface aims to provide a lightweight application that allows biologists to keep up with their experiments from anywhere (i.e. biologists can start, stop, and monitor their experiments from the internet.) Figure 8(b) shows a screen shot of the website where biologists can view the original microscope images, tracking results, and graphs of important cell metrics. The tracking results are saved as XML files and they are dynamically plotted onto the original microscope images by PHP. Through a combination of PHP and Javascript, users can view the result image sequence as a movie.

\section{Biological Applications}

We use two examples here to demonstrate how our cell image analysis system can facilitate applications in biology research.

\subsection{Wound Healing Assays}

The wound healing assay in vitro is widely used for research and discovery in biology and medicine. Figure 9(a) shows an example where cells on the edges of an artificial wound migrate toward the wound area. Our cell tracking system can track individual cells during the process and provide detailed spatiotemporal measurements of cell behaviors. For example, we can compute the decreasing size of the middle wound area based on tracking results (Figure 9(a)) and cell densities along image columns at each
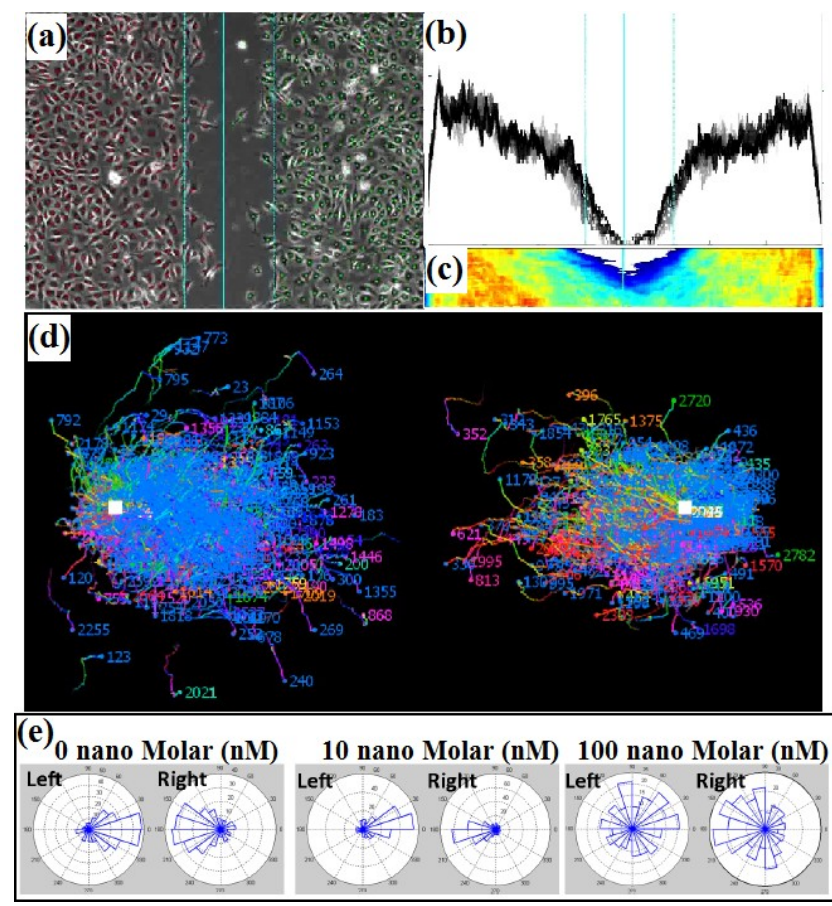

Figure 9. Wound healing assay. (a) Cells migrate to the middle wound area; (b) Cell densities along each image column; (c) Cell density map over time; (d) Cell migration paths (left and right groups); (e) Distributions of cell migration directions in response to different culturing conditions.

moment (Figure 9(b)). Figure 9(c) shows the jet map of cell density changes over time, each row of which represents the density curve values of Figure 9(b). Aligning all the cell migration paths with the same origin (white rectangles in Figure 9(d)), we can observe two clusters of movements where the left part of cells move rightward and vice versa. The above measurements are important for studying the influence of different culture conditions on the healing process. As shown in Figure 9(e), we plot the distribution of cell migration directions in rose diagrams. As the amount of medicine (Latrunculin B) increases, the bovine aortic endothelial cells tend to migrate more diversely.

\subsection{Stem Cell Manufacturing}

Stem cell research promises to revolutionize medicine. To meet the demand of clinical applications, a sufficient number of cells are needed during medical treatment (Figure 10(a)). Thus, cell culture conditions must maintain "stemness" for cell production, i.e., stem cells keep dividing without differentiating into fixed cell types (e.g. muscle, blood or skin cells). Quantifying metrics of stemness requires measuring the spatiotemporal histories of each cell's fate within a population. Given cell images captured in bio-labs, our system can track each individual cell's history (Figure 10(b)) and provide the full lineage trees (Figure $10(\mathrm{c}))$ to compute the metrics over time (e.g. cell pop- 

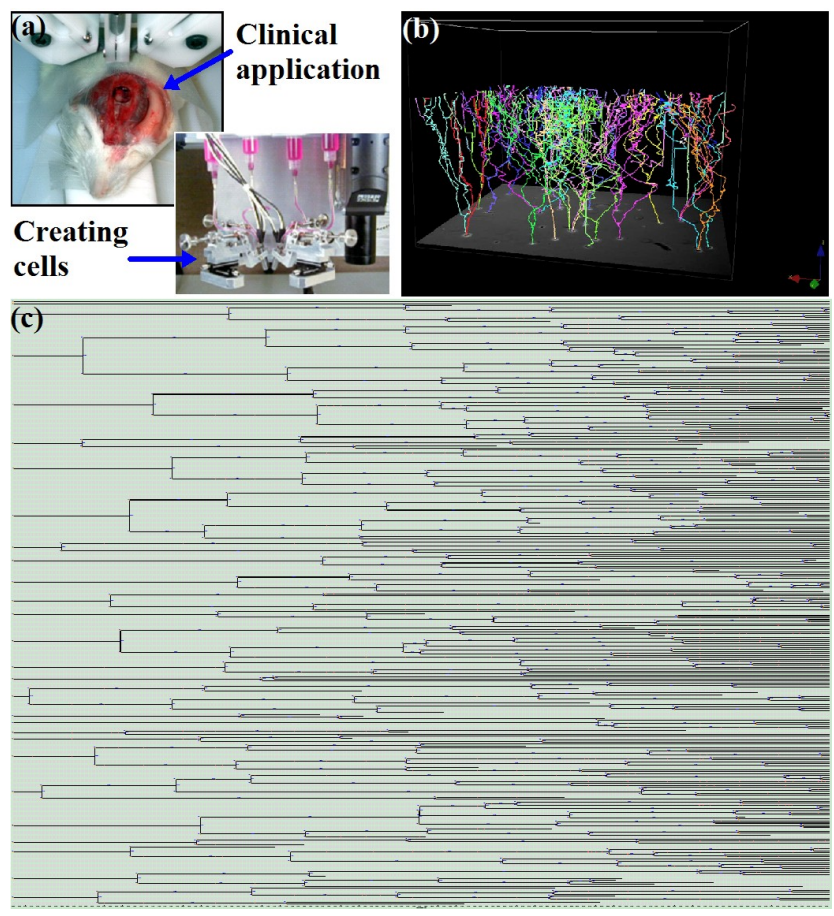

Figure 10. Stem cell manufacturing. (a) For clinical applications, enough numbers of stem cells are needed before medical treatment; (b) Tracked cell trajectories from captured image sequences; (c) The full cell lineage trees.

ulation, cell cycle, cell mitotic rate, tree synchrony etc.) Using these metrics, biologists can quantitatively and efficiently characterize cell behaviors in vitro in response to cell culturing conditions for maximizing cell expansion, even adaptively monitor and control in vivo medical therapy in the future.

\section{Conclusion}

We presented three cell image analysis algorithms: microscopy image restoration, mitosis event detection and error-tolerant data association, which are integrated into an online automated system. The system's performance is quantitatively evaluated on 16 sequences with promising accuracy. A public website compatible to a local GUI is set up as a community resource and run on a cloud computing cluster. Other research groups are welcome to upload their cell images for processing and comparison. We demonstrated the system's value on biology research using two biological applications: wound healing assay and stem cell manufacturing.

\section{References}

[1] O. Al-Kofahi et al., "Automated Cell Lineage Construction," Cell Cycle 5(3): 327-335, 2006. 2

[2] R. Bise, et al., "Reliably tracking partially overlapping neural stem cells in dic microscopy image sequences," MICCAI Workshop on OPTIMHisE, 2009. 5
[3] S. Blackman, "Multiple-Target Tracking with Radar Applications," Artech House Publishers, 1986. 6

[4] O. Debeir et al., "Tracking og Migrating Cells Under PhaseContrast Video Microscopy With Combined Mean-Shift Processes," IEEE Trans. on Medical Imaging, 24(6):697-711, 2005. 2

[5] O. Dzyubachyk et al., "Advanced Level-Set-Based Cell Tracking in Time-Lapse Fluorescence Microscopy," IEEE Trans. on Medical Imaging, 29(3):852-867, 2010. 2

[6] A. Hand et al., "Automated Tracking of Migrating Cells in Phase-contrast Video Microscopy Sequences using Image Registration,” Journal of Microscopy, 234: 62-79, 2009. 1, 2

[7] D. House et al., "Tracking of Cell Populations to Understand their Spatio-Temporal Behavior in Response to Physical stimuli," IEEE CVPR Workshop on Mathematical Modeling in Biomedical Image Analysis (MMBIA), 2009. 2

[8] K. Li et al., "Cell Population Tracking and Lineage Construction with Spatiotemporal Context," Medical Image Analysis, 12(5):546-566, 2008. 2, 6

[9] K. Li et al., "Computer Vision Tracking of Stemness," IEEE International Symposium on Biomedical Imaging (ISBI), 2008. 2

[10] K. Li and T. Kanade, "Nonnegative Mixed-Norm Preconditioning for Microscopy Image Segmentation," Proceedings of IPMI, 2009. 3

[11] A. Levin et al., "A Closed Form Solution to Natural Image Matting," IEEE Trans. on Pattern Analysis and Machine Intelligence, 30(2): p228-p242, 2008. 3

[12] A. Liu et al., "Mitosis Sequence Detection using Hidden Conditional Random Fields," IEEE International Symposium on Biomedical Imaging (ISBI), 2010. 2

[13] D. Lowe, "Object Recognition from Local Scale-invariant Features," IEEE International Conference on Computer Vision, 1999. 3

[14] E. Meijering et al., "Tracking in Cell and Developmental Biology," Seminars in Cell and Developmental Biology, 20(8): 894-902, 2009. 1, 2

[15] D. Padfield et al., "Coupled Minimum-Cost Flow Cell Tracking," International Conference on Information Processing in Medical Imaging (IPMI), 2009. 2

[16] A. Quattoni et al., "Hidden Conditional Random Fields," IEEE Trans. on Pattern Analysis and Machine Intelligence (PAMI), 29(10): 1848-1853, 2007. 4

[17] J. Rittscher, "Characterization of Biological Processes through Automated Image Analysis," Annual Review of Biomedical Engineering, 12: 315-344, 2010. 1

[18] F. Sha et al., "Multiplicative Updates for Nonnegative Quadratic Programming," Neural Computation, 19(8):20042031, 2007. 3

[19] I. Smal et al., "Particle Filtering for Multiple Object Tracking in Dynamic Fluorescence Microscopy Images: Application to Microtubule Growth Analysis," IEEE Trans. on Medical Imaging, 27(6): 789-804, 2008. 1

[20] F. Yang et al., "Cell Segmentation, Tracking, and Mitosis Detection Using Temporal Context," Proceedings of MICCAI 2005. 2

[21] Z. Yin et al., "Understanding the Optics to Aid Microscopy Image Segmentation,” Procceedings of MICCAI, 2010. 3 